\title{
Field-induced dissociation of electron-hole pairs in organic light emitting diodes monitored directly from bias-dependent magnetic resonance techniques
}

\section{Katsuichi Kanemoto, Shuto Hatanaka, Keigo Kimura, Yujiro Ueda, Hidenobu Matsuoka}

\begin{tabular}{|c|l|}
\hline Citation & Physical Review Materials, 1(2);022601 \\
\hline Issue Date & 2017-07-24 \\
\hline Type & Journal Article \\
\hline Textversion & Publisher \\
\hline \multirow{3}{*}{ Rights } & $\begin{array}{l}\text { CAmerican Physical Society. This article may be downloaded for personal use } \\
\text { only. Any other use requires prior permission of the author and American Physical } \\
\text { Society. } \\
\text { The following article appeared in Physical Review Materials Vol.1, Iss.2 and may } \\
\text { be found at https://doi.org/10.1103/PhysRevMaterials.1.022601 }\end{array}$ \\
\hline DOI & \begin{tabular}{l} 
10.1103/PhysRevMaterials.1.022601 \\
\hline
\end{tabular} \\
\hline
\end{tabular}

\author{
Self-Archiving by Author(s) \\ Placed on: Osaka City University
}

KANEMOTO, K., HATANAKA, S., KIMURA, K., UEDA, Y., \& MATSUOKA, H. (2017). Field-induced dissociation of electron-hole pairs in organic light emitting diodes monitored directly from bias-dependent magnetic resonance techniques. Physical Review Materials. 1. 022601. 


\title{
Field-induced dissociation of electron-hole pairs in organic light emitting diodes monitored directly from bias-dependent magnetic resonance techniques
}

\author{
Katsuichi Kanemoto, Shuto Hatanaka, Keigo Kimura, Yujiro Ueda, and Hidenobu Matsuoka \\ Department of Physics, Graduate School of Science, Osaka City University, 3-3-138 Sugimoto, Sumiyoshi-ku, Osaka 558-8585, Japan
}

(Received 25 November 2016; revised manuscript received 23 June 2017; published 24 July 2017)

\begin{abstract}
The conversion processes of electron-hole (e-h) pairs into carriers and excitons in organic light emitting diodes (OLEDs) have been explored by simultaneous photoluminescence- and photocurrent-detected magnetic resonance (PLDMR and PCDMR) techniques as a function of bias. Both PLDMR and PCDMR signals are shown to disappear or extremely reduce with increasing the field strength, whereas photoluminescence intensity is unchanged and photocurrent rather increases. This is clear evidence that e-h pairs are dissociated by the field. A simple model for pair dissociation is proposed and explains the field dependence of PLDMR intensity.
\end{abstract}

DOI: 10.1103/PhysRevMaterials.1.022601

Highly efficient organic light emitting diodes (OLEDs) have recently been developed by exploiting thermal transfer processes from triplet excitons (TEs) to singlet excitons (SEs) $[1,2]$. Such exploitation of correlated electronic states with different spin multiplicity is a recent challenging topic and understanding physics of the conversion process between the different spin-multiplicity states is currently required. The excitons initially generate as electron-hole (e-h) pairs and the e-h pairs play significant roles in OLED operation as intermediate states between carriers and excitons. Importantly, the pairs are responsible for prominent magnetic field effects (MFEs) of OLEDs such as magnetoresistance and magnetoelectroluminescence [3-11] and have recently been exploited as quantum sources because they may form entangled quantum states between the spin sublevels of singlet and triplet e-h pairs (SPs and TPs) [12-15]. Despite the significant contributions to such diverse fields, behaviors of e-h pairs under electric field has not been well explored in considering the mechanisms of OLED operation and MFE. The insufficient exploration corresponds to implicitly assuming the e-h pairs to generate simply in proportion to the carrier or exciton density and neglecting bias-dependent changes in conversion processes of e-h pairs into carriers and excitons. The reason for the insufficient exploration is precisely because methods of monitoring the e-h pair conversion in operating devices are unestablished.

For probing the e-h pairs, conventional electron spin resonance (ESR) techniques measuring microwave absorption would be inapplicable because coexistent stronger signals from carriers and defects typically overwhelm signals from e-h pairs. We thus here focus on pair-sensitive changes in equilibrium balance between SP and TP induced by the ESR transition. It can be monitored via slight changes of emission intensity and/or current $[12,16]$. However, interpretations of such ESR-induced signals have been controversial $[16,17]$ and simple use of the techniques cannot offer convincing data as the pair-related phenomena. Effective ESR methods for probing behaviors of pairs thus need to be developed.

In this Rapid Communication, the conversion processes of e-h pairs into carriers and excitons in OLEDs are addressed by current- and emission-detection techniques of ESR. The conversion process would depend largely on the electric-field

*Corresponding author: kkane@sci.osaka-cu.ac.jp strength, and hence the measurements were made as a function of bias. Under OLED operating conditions, however, the effect of bias on the e-h pairs is almost dominated by a bias-dependent pair generation process, making it difficult to monitor fielddependent behaviors of pairs. We thus designed the ESR experiments combined with photoexcitation: photoluminescence (PL) -detected and photocurrent (PC) -detected magnetic resonance (PLDMR and PCDMR, respectively), under a bias region of negligible carrier injection from electrodes for polymer OLEDs. The PLDMR and PCDMR experiments performed simultaneously indeed enable observation of fielddependent conversion processes of e-h pairs: recombination and dissociation into excitons and carriers, respectively. The e-h pairs are found to disappear under internal field above $0.5 \mathrm{MV} / \mathrm{cm}$. Such field effects on e-h pairs would influence the MFE and operation mechanisms of OLEDs. We propose bias-dependent magnetic resonance techniques as monitoring tools effective for the e-h pairs under electric field.

Two types of poly(-para-phenylene vinylene) (PPV) derivatives were used for the active polymer layer of diodes: poly[2-methoxy-5-(2'-ethylhexyloxy)-para-phenylene vinylene] (MEH-PPV) and super yellow (SY) PPV purchased from Aldrich and Merck, respectively. The polymer layer was fabricated by spin casting the chlorobenzene solution $(5 \mathrm{mg} / \mathrm{ml})$ on the molybdenum trioxide layer $(20 \mathrm{~nm})$ vacuum deposited on the indium-tin-oxide (ITO) coated glass substrate (anode). A polyethyleneimine (PEI) layer was spin casted on the PPV layer from the ethanol solution $(80 \mu \mathrm{g} / \mathrm{ml})$ and an Al layer was finally vacuum deposited for the cathode. The total thickness of the PPV layer including the PEI layer was about $50 \mathrm{~nm}$ for MEH-PPV and $100 \mathrm{~nm}$ for SY-PPV. Both diodes exhibited clear electroluminescence under forward bias over around $4 \mathrm{~V}$. A continuous-wave diode laser at $473 \mathrm{~nm}\left(640 \mathrm{~mW} / \mathrm{cm}^{2}\right)$ was used for photoexcitation. PL intensity and PC were measured using lock-in techniques synchronized with the laser output chopped at $700 \mathrm{~Hz}$. PLDMR and PCDMR measurements were performed by recording lock-in signals of the PL and PC signals under continuous photoexcitation synchronized with the microwave modulation $(70 \mathrm{~mW}, 1000 \mathrm{~Hz})$. The ESR response from dark current and the off-resonance signal induced by the microwave modulation were negligibly small or eliminated. All measurements were performed at room temperature under nitrogen gas flow. 


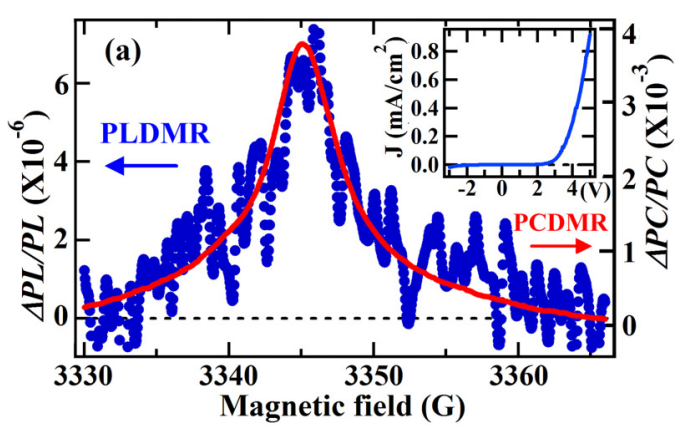

(b)

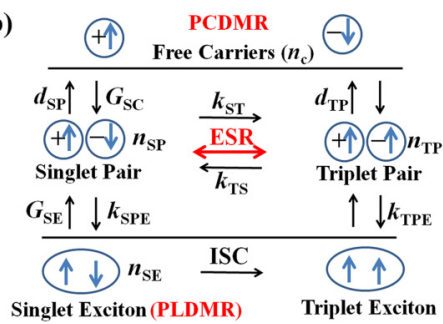

FIG. 1. (a) Spectra of PLDMR and PCDMR for SY-PPV OLED measured simultaneously at zero-bias voltage. The inset shows current-voltage characteristics of the OLED. (b) Equilibrium relation expected among carriers, electron-hole pairs, and excitons. The ESR transition is expected to occur at the sublevels of triplet pairs, causing equilibrium shifts between singlet and triplet pairs.

Figure 1(a) shows the PLDMR and PCDMR spectra of the SY-PPV diode (SY diode) measured simultaneously at zerobias voltage. The spectra of PLDMR and PCDMR resemble each other and have a half-width of about $6.9 \mathrm{G}$. This spectral resemblance was also observed in the MEH-PPV OLED (MEH-LED) (Supplemental Material [18]) and particularly invariably identified from different batch diodes even when the magnitude of half-width was different. Thus, spin species responsible for PLDMR and PCDMR signals are common to each diode. In addition, the PCDMR spectra of the two diodes could be fitted by the sum of two Gaussian curves [18]. The two components are explainable as resulting from electrons and holes of e-h pairs [19]. These signals are thus suggested to result from the spin transition at e-h pairs.

The mechanism of PLDMR and PCDMR signals in polymer diodes has been discussed in several references $[16,17,20,21]$. Shinar and co-workers regarded spin-dependent reactions between TEs and polarons as relevant to the PLDMR mechanism [17,22]. However, the intersystem crossing from SE to TE in MEH-PPV was predicted to be inefficient [23,24]. The photogeneration efficiency of TE should thus be low in the MEH diode, suggesting minor contributions of TE to PLDMR signals. Therefore, considering the observed resemblance in PLDMR and PCDMR spectra common to the two diodes, we regard spin-dependent reactions (SDRs) at e-h pairs as responsible for PLDMR and PCDMR signals in both SY and MEH- diodes.

In the model of SDRs [12,16], an equilibrium relation shown in Fig. 1(b) is assumed among free polaron carriers, e-h pairs, and excitons. The free carriers are here provided directly from phototransition without dissociation of excitons. Such direct photocarrier generation is justified by the presence of photocurrent in the short circuit condition, which was indeed identified from the present diodes. For this system, the steady-state PL intensity $I_{\mathrm{PL}}$, proportional to the density of $\mathrm{SE}\left(n_{\mathrm{SE}}\right)$, is calculated as follows from the solution of rate equation:

$$
I_{\mathrm{PL}} \propto n_{\mathrm{SE}}=\left(G_{\mathrm{E}}+k_{\mathrm{SPE}} n_{\mathrm{SP}}\right) \tau_{\mathrm{PL}} \approx G_{\mathrm{E}} \tau_{\mathrm{PL}},
$$

where $G_{\mathrm{E}}$ is the generation density of SE by photoexcitation including the photoexcitation intensity, $k_{\mathrm{SPE}}$ the transition rate of SP to SE, $n_{\mathrm{SP}}$ the density of SP, and $\tau_{\mathrm{PL}}$ the PL lifetime. For this equilibrium balance, the ESR transition occurring at the spin sublevels of TP modulates $n_{\mathrm{SP}}$, resulting in generation of PLDMR signals according to the next relation

$$
\begin{aligned}
I_{\mathrm{PLDMR}} \propto \Delta_{\mathrm{ESR}}\left(n_{\mathrm{SE}}\right) & =\Delta_{\mathrm{ESR}}\left(k_{\mathrm{SPE}} n_{\mathrm{SP}} \tau_{\mathrm{PL}}\right) \\
& =k_{\mathrm{SPE}} \tau_{\mathrm{PL}} \Delta_{\mathrm{ESR}}\left(n_{\mathrm{SP}}\right),
\end{aligned}
$$

where $\Delta_{\text {ESR }}$ indicates a variation of each term at the ESR transition. We observed positive PLDMR signals from both diodes, indicating equilibrium shifts occurring from TP to SP by the ESR transition $\left[\Delta_{\mathrm{ESR}}\left(n_{\mathrm{SP}}\right)>0\right]$. SPs and TPs are expected to dissociate into free carriers with the dissociation rates $d_{\mathrm{SP}}$ and $d_{\mathrm{TP}}$, respectively. A slight difference between $d_{\mathrm{SP}}$ and $d_{\mathrm{TP}}$ can result in changing a current density $J\left(=e n_{c} \mu F\right)$ when the equilibrium from TP to SP shifts by the ESR transition. The PCDMR intensity is then given as follows:

$$
I_{\mathrm{PCDMR}}=\Delta_{\mathrm{ESR}}(J)=e \mu F \Delta_{\mathrm{ESR}}\left(n_{c}\right),
$$

where $n_{c}$ is the carrier density, $\mu$ the carrier mobility, and $F$ the strength of internal electric field. We expect this PCDMR mechanism to be dominant in the present diodes. Positive PCDMR signals were observed, suggesting a relation $d_{\mathrm{SP}}>d_{\mathrm{TP}}$, according to the increased $n_{\mathrm{SP}}$ identified from the PLDMR results.

In the equilibrium relation in Fig. 1(b), the dissociation rates into carriers and transition rates of the e-h pairs into excitons can vary depending on external voltages. The current-voltage characteristics of the SY diode in the inset of Fig. 1(a) show carrier injection from the electrodes to be negligible below $2.5 \mathrm{~V}$ in the dark condition. e-h pairs are thus supplied only from photoexcitation and we expect the density of photogenerated pairs to be nearly bias independent. Hence, research of bias-dependent dissociation of the e-h pairs is possible under bias below $2.5 \mathrm{~V}$.

Figures 2(a) and 2(b) show the voltage dependences of PLDMR and PCDMR signals in the SY diode, respectively, measured from $-3 \mathrm{~V}$ to $3 \mathrm{~V}$ at the resonant peak (3345 G). The PLDMR intensity has a maximum around $2.2 \mathrm{~V}$ and is rapidly reduced by decreasing the bias and finally disappears around $-1 \mathrm{~V}$, whereas the PL intensity shown in the inset varies only slightly. Similarly to this trend, the PLDMR signals of the MEH diode shown in Fig. 2(c) exhibit a clear maximum peak around $2.2 \mathrm{~V}$ and disappeared around $-2 \mathrm{~V}$. The maximum voltage is close to the rise point of dark current and corresponds to the built-in voltage $V_{\mathrm{bi}}$ of the diodes where $F$ is nulled (Supplemental Material [18]). Moreover, the voltage is close to the zero point of PC shown in the inset of Fig. 2(b), although the zero point could deviate slightly from an exact zero PC point due to space charge effects from carriers. These features demonstrate PLDMR signals to be extremely $F$ dependent. The PCDMR signals of SY-PPV 

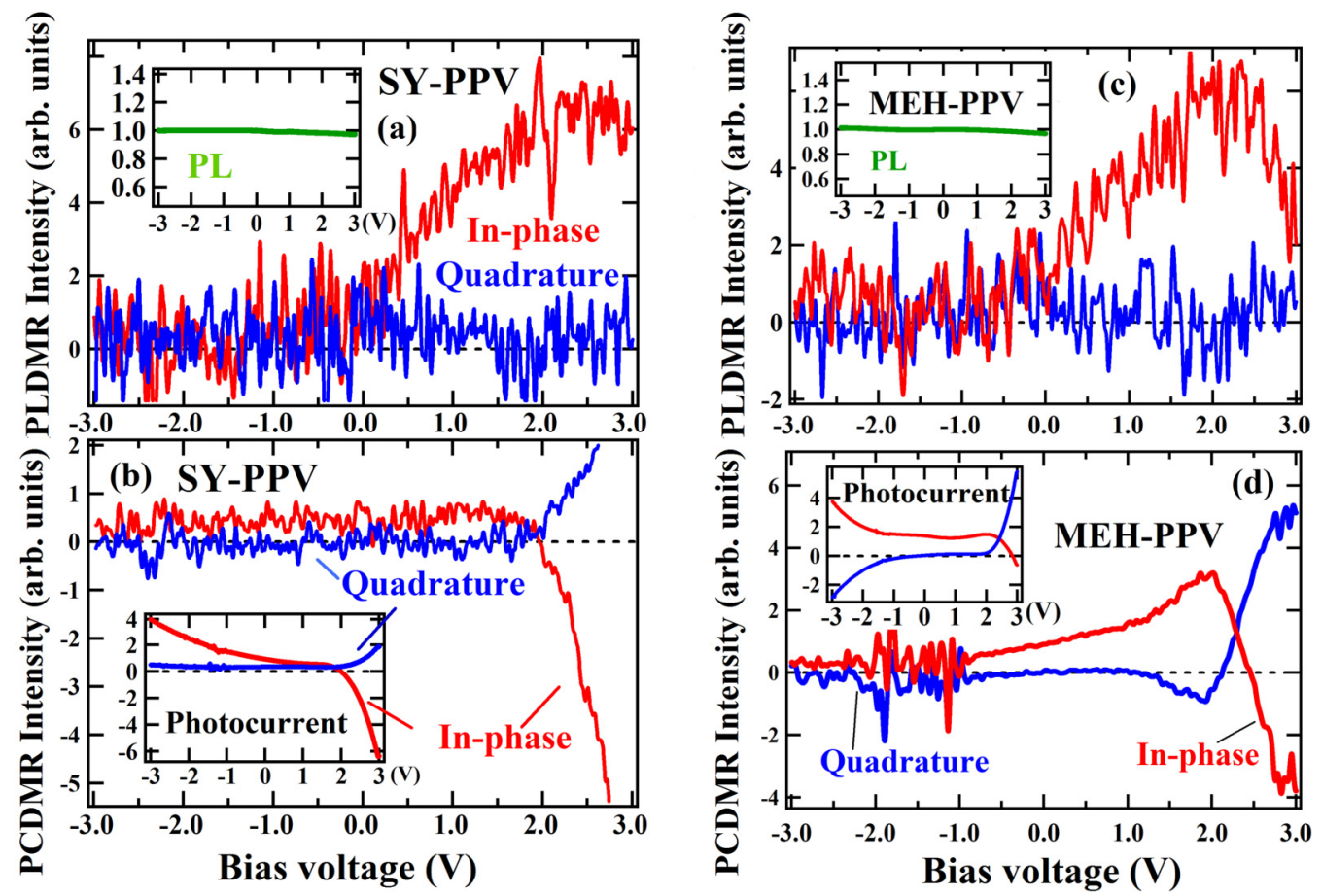

FIG. 2. Bias dependence of the PLDMR (a) and PCDMR (b) intensities measured simultaneously for the SY-PPV diode. The inset shows bias dependence of PL intensity (a) and photocurrent (b) measured simultaneously for the same diode. (c) and (d) show the results of the same experiments for the MEH-PPV diode as that in (a) and (b), respectively.

have a quadrature lock-in component with different current characteristics from the in-phase component and the main PCDMR signals are given by the in-phase component. Similar two-phase characteristics are also found from the PC result shown in the inset. Importantly, the main PCDMR signals are weakly reduced by decreasing the voltage from $1 \mathrm{~V}$, whereas the PC magnitude increases in the same voltage range. Similar but clearer trends are identified from the MEH- diode shown in Fig. 2(d). The reduction or disappearance observed both in the PLDMR and PCDMR signals indicates that e-h pairs transferring into carriers and excitons are absent in the diodes under a strong electric-field magnitude. These observations are evidence of field-induced dissociation occurring in the e-h pairs.

Features of the field-induced dissociation of e-h pairs observed in PLDMR and PCDMR signals are here discussed. According to Eq. (2), the field dependence of PLDMR signals is determined by the following factors:

$$
I_{\mathrm{PLDMR}}(F) \propto \tau_{\mathrm{PL}} k_{\mathrm{SPE}}(F) \Delta_{\mathrm{ESR}}\left[n_{\mathrm{SP}}(F)\right],
$$

where a change of $\tau_{\mathrm{PL}}$ at the ESR transition is neglected. The two factors, $k_{\mathrm{SPE}}$ and $\Delta_{\mathrm{ESR}}\left(n_{\mathrm{SP}}\right)$, might change depending on $F$. The field dependence of PCDMR signals can also be considered from Eq. (3). However, it includes $\mu$ and $F$, and the field dependence of $\mu$ is unclear. For eliminating contributions from the two field-dependent factors, $\Delta_{\mathrm{ESR}}(J)$ divided by $J$ is considered as follows:

$$
\Delta_{\mathrm{ESR}}(J) / J=\Delta_{\mathrm{ESR}}\left(n_{c}\right) / n_{c} .
$$

$n_{c}$ and $\Delta_{\mathrm{ESR}}\left(n_{c}\right)$ are here obtained by

$$
\begin{aligned}
n_{c} & =\left(G_{c}+d_{\mathrm{SP}} n_{\mathrm{SP}}+d_{\mathrm{TP}} n_{\mathrm{TP}}\right) \tau_{c} \approx G_{c} \tau_{c}, \\
\Delta_{\mathrm{ESR}}\left(n_{c}\right) & =\left\{d_{\mathrm{SP}} \Delta_{\mathrm{ESR}}\left(n_{\mathrm{SP}}\right)+d_{\mathrm{TP}} \Delta_{\mathrm{ESR}}\left(n_{\mathrm{TP}}\right)\right\} \tau_{c},
\end{aligned}
$$

where $G_{c}$ is the generation density of photocarriers and $\tau_{c}$ the carrier lifetime. Here the following relation holds between $\Delta_{\mathrm{ESR}}\left(n_{\mathrm{SP}}\right)$ and $\Delta_{\mathrm{ESR}}\left(n_{\mathrm{TP}}\right)[18]$ :

$$
\left(d_{\mathrm{SP}}+k_{\mathrm{SPE}}\right) \Delta_{\mathrm{ESR}}\left(n_{\mathrm{SP}}\right)+\left(d_{\mathrm{TP}}+k_{\mathrm{TPE}}\right) \Delta_{\mathrm{ESR}}\left(n_{\mathrm{TP}}\right)=0 .
$$

Considering these relations, we obtain the field dependence of the normalized PCDMR intensity as follows:

$$
\begin{aligned}
& \frac{\Delta_{\mathrm{ESR}}[J(F)]}{J(F)} \\
& \quad=\frac{d_{\mathrm{SP}}(F) k_{\mathrm{TPE}}(F)-d_{\mathrm{TP}}(F) k_{\mathrm{SPE}}(F)}{G_{c}\left[d_{\mathrm{TP}}(F)+k_{\mathrm{TPE}}(F)\right]} \Delta_{\mathrm{ESR}}\left[n_{\mathrm{SP}}(F)\right] .
\end{aligned}
$$

Both the field dependence of the PLDMR intensity and the normalized PCDMR intensity calculated in Eqs. (4) and (8) include the factor $\Delta_{\mathrm{ESR}}\left[n_{\mathrm{SP}}(F)\right] . \Delta_{\mathrm{ESR}}\left[n_{\mathrm{SP}}(F)\right]$ is expected to be proportional to the total pair density $n_{\mathrm{SP}}(F)+n_{\mathrm{TP}}(F)$. Hence both of the intensities reduce when the pairs are field dissociated. Figures 3(a) and 3(b) display the field dependence of the PLDMR intensity and the normalized PCDMR intensity calculated for the SY diode and $\mathrm{MEH}$ diode considering each film thickness. In both diodes, the PLDMR and PCDMR results exhibit similar field-dependent curves, suggesting that the term of $\Delta_{\mathrm{ESR}}\left[n_{\mathrm{SP}}(F)\right]$ dominates both results and that they are determined by the field-dependent dissociation of e-h pairs.

Field-dependent dissociation of charge pairs has often been analyzed by the Onsager-Braun model $[25,26]$. The model was 

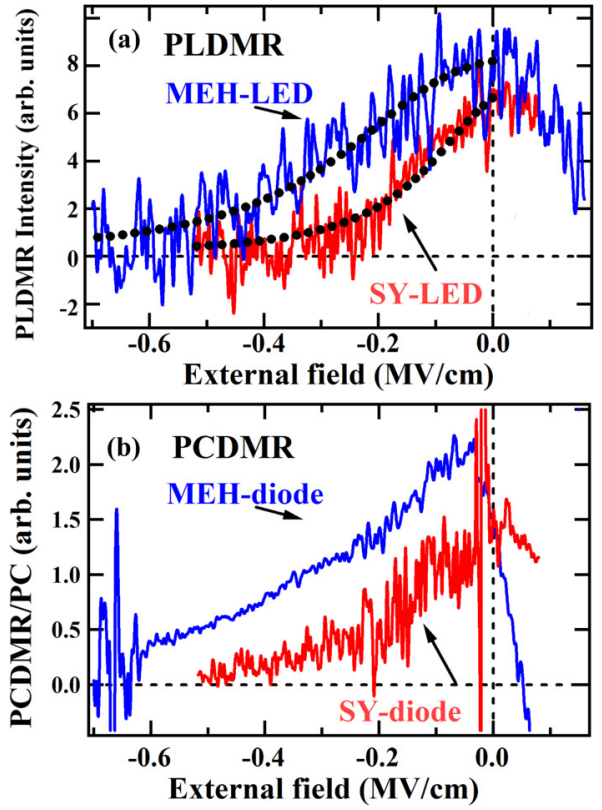

(c)
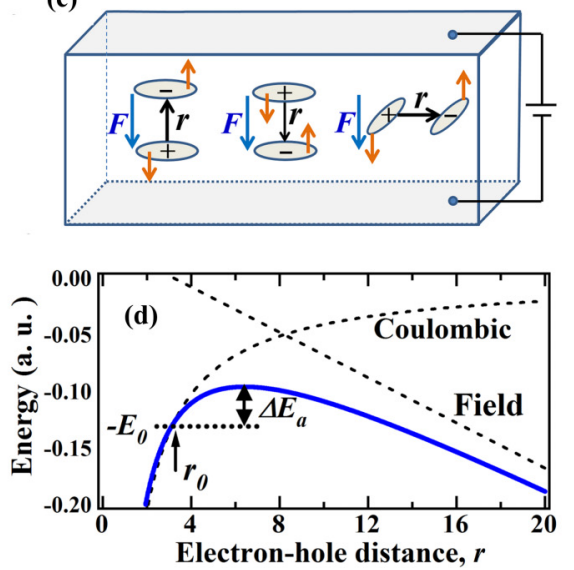

FIG. 3. (a) Field dependence of PLDMR signals in SY-PPV and MEH-PPV diodes. Dotted lines indicate the results of fit calculated using Eq. (11). (b) Field dependence of the normalized PCDMR signals in SY-PPV and MEH-PPV diodes. (c) Three examples of orientation of the pair dipole vector $\boldsymbol{r}$ against the external field $\boldsymbol{F}$. (d) Schematic of the model used for analyzing the fieldinduced dissociation of electron-hole pairs. The activation energy for dissociation $\Delta E_{a}$ is reduced by increasing electric field. $E_{0}$ is the Coulomb binding energies between electrons and holes and $r_{0}$ the electron-hole distance of e-h pairs under zero external field.

first built for charge pairs in solvent [25], and shown to explain field-dependent dissociation of charge-transfer pairs photogenerated in organic crystals [27]. It could thus be applicable to e-h pairs in this system. However, the Onsager-Braun model assumes free diffusion of charge pairs in quasicontinuum as in solvent, but such free diffusions could be unsuitable for e-h pairs dispersed in the amorphous polymer film. Alternatively, a model treating local field-induced transfer processes of electrons as in Poole-Frenkel type [28] could be better for such non-free electron transfers expected in the e-h pairs. We thus hereafter attempt to explain the bias-dependent ESR signals using a modified Poole-Frenkel model.
We first consider the effect of the direction of the dipole vector of the e-h pair against the external field as illustrated in Fig. 3(c). When the vector and field are antiparallel to each other, the field promotes pair dissociation and surviving pairs contribute to PLDMR or PCDMR signals. In the case of parallel configuration, the field may assist recombination of pairs, leading to emission. Indeed, the presence of field-assist recombination was recently proposed for the electroluminescence (EL) process of OLEDs [24,29]. We thus regard the parallel configuration as being assisted by the field. The influence of the field perpendicular to the dipole vector should be carefully treated. In the case of classical dipoles, they rotate for a perpendicular field while keeping the electron-hole distance. However, such a rotation is unlikely to occur in e-h pairs because the rotation needs continuous transfer of electrons and holes inside the diode. Instead, the perpendicular field would induce the transfer of holes and/or electrons in pairs into adjacent chains along the field direction, resulting in pair dissociation. Actually, the observations of PLDMR signals disappearing completely by increasing external fields are the evidence that the perpendicular components of pairs are also field dissociated without surviving under the field.

From these considerations, the field-induced pair reduction is expected to occur at all directions. The pair reduction would also depend on many elements such as the electron-hole distance, the twist angle of polymer chains where holes and electrons reside, and their morphological distribution. Considering all these elements is virtually impossible experimentally and theoretically. We thus treat a simple model where the field-induced dissociation and recombination are assumed to occur isotropically with the same field dependence both in SPs and in TPs. We also neglect distributions of the electron-hole distance for simplicity.

We here regard the pair dissociation and recombination as starting with transfers of holes into adjacent chains under the influences of external-field and Coulomb potential by electrons. Under this condition, the activation energy of the transfer is reduced with increasing external fields [Fig. 3(d)] (Supplemental Material [18]). The probability of pair dissociation and recombination $\phi(F)$ is then obtained as follows:

$$
\begin{aligned}
\phi(F) & =\exp \left\{\frac{-\Delta E_{a}(F)}{k_{B} T}\right\} \\
& =\exp \left\{\frac{-\beta^{2} /\left(4 e r_{0}\right)+\beta \sqrt{|F|}-e r_{0}|F|}{k_{B} T}\right\},
\end{aligned}
$$

where $\Delta E_{a}(F)$ is the field-dependent activation energy, $\beta=\left(e^{3} / \pi \varepsilon \varepsilon_{0}\right)^{1 / 2}, k_{B}$ is the Boltzmann constant, $T$ is the temperature, and $r_{0}$ is the electron-hole distance of e-h pairs under zero external field. $\phi(F)$ enhances the dissociation and recombination rates and we assume the following relation for, e.g., $d_{s p}$ :

$$
d_{\mathrm{SP}}=d_{\mathrm{SP}, 0}\left\{\phi(F)+\phi_{0}\right\}
$$

where $d_{\mathrm{SP}, 0}$ is the proportionality factor. Also $\phi_{0}$ is the zerofield factor and was employed to represent the contribution of dissociation or recombination at zero field. By this treatment, the probability of dissociation or recombination at $F$ is given by $\left\{\phi(F)+\phi_{0}\right\} /\left(1+\phi_{0}\right)$. 
From the above treatments, we attempt to fit the field dependence of PLDMR signals for the SY-LED and MEH-LED shown in Figs. 3(a) and 3(b). In reality, under the assumption of isotropic field-induced dissociation and recombination, $I_{\text {PLDMR }}(F)$ in Eq. (4) is obtained by [18]

$$
I_{\operatorname{PLDMR}}(F) \propto\left\{\phi(F)+\phi_{0}\right\}^{-1} .
$$

Using this relation, the PLDMR results were fitted with $V_{\mathrm{bi}}(=2.2 \mathrm{~V})$ with $\varepsilon=3.5$. For the same parameter of $\phi_{0}=$ $0.09, r_{0}$ was determined as being 2.3 and $1.8 \mathrm{~nm}$ for the SYLED and MEH-LED, respectively. The Coulomb binding energies between electrons and holes of e-h pairs, $E_{0}=\beta^{2} /\left(4 e r_{0}\right)$, are then calculated to be 0.18 and $0.23 \mathrm{eV}$, respectively. These values were obtained by neglecting distribution of $r_{0}$, although consideration of the distribution would influence the determined $r_{0}$ values [27,30]. Nevertheless, the $r_{0}$ values are close to the average e-h distance of poly(styrene-sulfonate)-doped poly(3,4-ethylenedioxythiophene) (PEDOT:PSS), $2.1 \mathrm{~nm}$, estimated by analyzing Rabi oscillations of electrical ESR signals from pulsed microwave irradiation [15]. In addition, the interlamellar distance of poly(3-alkylthiophenes) was previously shown to depend on the length of alkyl side chain [31]. The obtained $r_{0}$ values $(1.8$ and $2.3 \mathrm{~nm})$ are close to the interlamellar distance for the cases of $\mathrm{C}_{6} \mathrm{H}_{13}(1.64 \mathrm{~nm})$, $\mathrm{C}_{8} \mathrm{H}_{17}(2.01 \mathrm{~nm})$, and $\mathrm{C}_{10} \mathrm{H}_{21}(2.39 \mathrm{~nm})$ side chains [31]. We thus predict that the differences in the bulkiness and length of side chains could determine the difference in the e-h distance between the SY diode and the MEH diode.

The relative comparison of e-h pair distance (the pairbinding energy) between the two diodes was made by assuming that spatial pair distributions inside the diode are similar. Such comparison should also be possible between the e-h pair and SE for each diode because their distribution in diodes would be similar. The field-induced dissociation of SE can be monitored from bias-dependent reductions in PL intensity [32-36]. Weak $\mathrm{PL}$ reductions were actually observed below $-6 \mathrm{~V}$ as shown in Fig. S6 [18]. The much higher bias magnitude for SE dissociation than that for the e-h pair is well consistent with the exciton binding energy $(0.4-0.6 \mathrm{eV})$ estimated previously for conjugated polymers $[33,36]$, suggesting relevance of the estimated binding energy of e-h pairs.

These consequences confirm that bias-dependent PLDMR and PCDMR measurements are effective for exploring the stability of e-h pairs and their physical properties under electric field. Such properties of e-h pairs have been studied from a bias-dependent change in a photocurrent induced by pair dissociation [27]. However, the pair properties were derived from carriers via current but not directly obtained from pairs. The magnetic resonance techniques have definite advantages for studies of pair properties because they are directly obtained from spin transitions of the pairs. Moreover, the magnetic resonance experiments can be performed simultaneously from optical and electrical detection techniques,

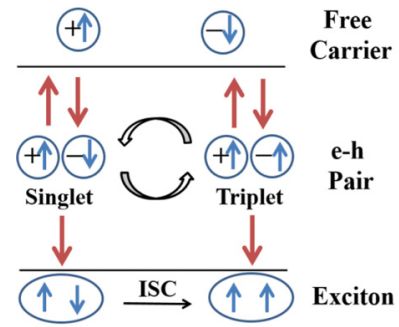

(a) $|F|<0.5 \mathrm{MV} / \mathrm{cm}$

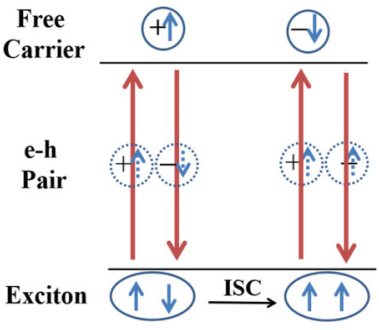

(b) $|F|>0.5 \mathrm{MV} / \mathrm{cm}$
FIG. 4. Schematics of equilibrium shifts occurring among carriers, electron-hole pairs, and excitons under the field strength $<0.5 \mathrm{MV} / \mathrm{cm}$ (a) and $>0.5 \mathrm{MV} / \mathrm{cm}$ (b). In the case of (b), the electron-hole pairs are virtually absent.

enabling research of e-h pairs both via excitons as well as carriers. In the Onsager-Braun model, the recombination rate of charge pairs into excitons is usually assumed to be field independent [26,30]. However, the recent reports suggesting field-dependent recombination processes of pairs in the EL process of OLEDs [24,29] indicate the necessity of studying field-dependent properties of e-h pairs from both carrier and exciton sides. The magnetic resonance techniques are currently the only techniques enabling the research.

Finally, the effect of electric field on e-h pairs is summarized in Fig. 4. Under a weak field, e-h pairs are created only from free carriers because excitons are not field dissociated, and e-h pairs can then dissociate or recombine depending on the field strength. Under a strong field typically over $0.5 \mathrm{MV} / \mathrm{cm}$, e-h pairs are unlikely to exist and direct conversions probably occur between free carriers and excitons. In this field range, MFEs responsible for the pairs could be reduced. However, we note that, although the present research was made for the bias region where charge injection from electrodes is negligible, injected carriers may screen electric fields inside diodes under large forward biases $[37,38]$. Therefore behaviors of e-h pairs under the forward bias region should be carefully examined and research for the bias region is currently underway.

In summary, the PLDMR and PCDMR signals of OLEDs were shown to extremely reduce with increasing the internal field, and the reduction was found to result from the dissociation of e-h pairs. The field dependence of the PLDMR and PCDMR signals was shown to differ between the SY diode and the MEH diode, being attributed to the difference of the electron-hole distance. These magnetic resonance techniques are effective to monitor field dissociation of e-h pairs and can be employed to consider the MFE and operation mechanism of OLEDs.

This work was supported in part by a Grant-in-Aid (Grant No. 25288117) from the Japanese Ministry of Education, Culture, Sports, Science, and Technology.
[1] H. Uoyama, K. Goushi, K. Shizu, H. Nomura, and C. Adachi, Nature (London) 492, 234 (2012).

[2] Q. S. Zhang, B. Li, S. P. Huang, H. Nomura, H. Tanaka, and C. Adachi, Nat. Photonics 8, 326 (2014).
[3] F. J. Wang, H. Bässler, and Z. Valy Vardeny, Phys. Rev. Lett. 101, 236805 (2008)

[4] U. Niedermeier, M. Vieth, R. Patzold, W. Sarfert, and H. von Seggern, Appl. Phys. Lett. 92, 193309 (2008). 
[5] S. A. Bagnich, U. Niedermeier, C. Melzer, W. Sarfert, and H. von Seggern, J. Appl. Phys. 106, 113702 (2009).

[6] T. D. Nguyen, G. Hukic-Markosian, F. J. Wang, L. Wojcik, X. G. Li, E. Ehrenfreund, and Z. V. Vardeny, Nat. Mater. 9, 345 (2010).

[7] T. D. Nguyen, B. R. Gautam, E. Ehrenfreund, and Z. V. Vardeny, Phys. Rev. Lett. 105, 166804 (2010).

[8] T. Ikoma, T. Ogiwara, Y. Takahashi, K. Akiyama, S. TeroKubota, T. Suzuki, and Y. Wakikawa, Synth. Met. 160, 285 (2010).

[9] F. Macia, F. J. Wang, N. J. Harmon, A. D. Kent, M. Wohlgenannt, and M. E. Flatte, Nat. Commun. 5, 3609 (2014).

[10] Y. C. Hsiao, T. Wu, M. X. Li, and B. Hu, Adv. Mater. 27, 2899 (2015).

[11] Y. F. Wang, K. Sahin-Tiras, N. J. Harmon, M. Wohlgenannt, and M. E. Flatte, Phys. Rev. X 6, 011011 (2016).

[12] D. R. McCamey, H. A. Seipel, S. Y. Paik, M. J. Walter, N. J. Borys, J. M. Lupton, and C. Boehme, Nat. Mater. 7, 723 (2008).

[13] D. R. McCamey, J. Van Tol, G. W. Morley, and C. Boehme, Science 330, 1652 (2010).

[14] W. J. Baker, T. L. Keevers, J. M. Lupton, D. R. McCamey, and C. Boehme, Phys. Rev. Lett. 108, 267601 (2012).

[15] K. J. van Schooten, D. L. Baird, M. E. Limes, J. M. Lupton, and C. Boehme, Nat. Commun. 6, 6688 (2015).

[16] S.-Y. Lee, S. Paik, D. R. McCamey, and C. Boehme, Phys. Rev. B 86, 115204 (2012).

[17] J. Shinar, Laser Photonics Rev. 6, 767 (2012).

[18] See Supplemental Material at http://link.aps.org/supplemental/ 10.1103/PhysRevMaterials.1.022601 for details.

[19] D. R. McCamey, K. J. van Schooten, W. J. Baker, S.-Y. Lee, S.-Y. Paik, J. M. Lupton, and C. Boehme, Phys. Rev. Lett. 104, 017601 (2010).

[20] A. Sperlich, H. Kraus, C. Deibel, H. Blok, J. Schmidt, and V. Dyakonov, J. Phys. Chem. B 115, 13513 (2011).
[21] K. Kanemoto, H. Matsuoka, Y. Ueda, K. Takemoto, K. Kimura, and H. Hashimoto, Phys. Rev. B 86, 125201 (2012).

[22] Y. Chen, M. Cai, E. Hellerich, R. Shinar, and J. Shinar, Phys. Rev. B 92, 115203 (2015).

[23] H. D. Burrows, J. S. de Melo, C. Serpa, L. G. Arnaut, M. D. Miguel, A. P. Monkman, I. Hamblett, and S. Navaratnam, Chem. Phys. 285, 3 (2002).

[24] T. Takahashi, K. Kanemoto, M. Kanenobu, Y. Okawauchi, and H. Hashimoto, Sci. Rep. 5, 15533 (2015).

[25] L. Onsager, Phys. Rev. 54, 554 (1938).

[26] C. L. Braun, J. Chem. Phys. 80, 4157 (1984).

[27] M. Pope and C. Swenberg, Electronic Processes in Organic Crystals and Polymers, 2nd ed. (Oxford University Press, Oxford, 1999), Chap. III.

[28] P. N. Murgatroyd, J. Phys. D 3, 151 (1970).

[29] S. Yin, L. Chen, P. Xuan, K. Q. Chen, and Z. Shuai, J. Phys. Chem. B 108, 9608 (2004).

[30] T. E. Goliber and J. H. Perlstein, J. Chem. Phys. 80, 4162 (1984).

[31] T. A. Chen, X. M. Wu, and R. D. Rieke, J. Am. Chem. Soc. 117, 233 (1995).

[32] R. Kersting, U. Lemmer, M. Deussen, H. J. Bakker, R. F. Mahrt, H. Kurz, V. I. Arkhipov, H. Bassler, and E. O. Gobel, Phys. Rev. Lett. 73, 1440 (1994).

[33] M. Deussen, P. H. Bolivar, G. Wegmann, H. Kurz, and H. Bassler, Chem. Phys. 207, 147 (1996).

[34] V. I. Arkhipov, E. V. Emelianova, and H. Bassler, Phys. Rev. Lett. 82, 1321 (1999).

[35] M. Segal, M. A. Baldo, R. J. Holmes, S. R. Forrest, and Z. G. Soos, Phys. Rev. B 68, 075211 (2003).

[36] H. Najafov, I. Biaggio, T. K. Chuang, and M. K. Hatalis, Phys. Rev. B 73, 125202 (2006).

[37] P. J. Brewer, P. A. Lane, A. J. deMello, D. D. C. Bradley, and J. C. deMello, Adv. Funct. Mater. 14, 562 (2004).

[38] K. Kanemoto, T. Takahashi, and H. Hashimoto, Appl. Phys. Lett. 109, 013301 (2016). 